\title{
A Study on the Circular Knitted Fabrics with Cosmetic Functions
}

\author{
FL Huang, JY Fu and KB Cheng* \\ Department of Fiber and Composite Materials, Feng Chia University, Taiwan
}

Submission: December 17, 2018; Published: January 10, 2019

*Corresponding author: Kou-Bin Cheng, Department of Fiber and Composite Materials, Feng Chia University, 100, Wenhua Road, Taichung 407, Taiwan

\begin{abstract}
The multi-functional and cosmetic fiber was developed in this paper which in response to the Kyoto Protocol and a number of international climate conference thinking regarding energy conservation, year-on-year reduction of carbon emissions and carbon. Using wet ball milling to grind, disperse, and modify the cooling mineral stone powder and extracting collagen, and then make caprolactam mix cooling powder and collagen filler through ring open reaction, and in-situ polymerization to fabricate nylon 6 slices with cooling powders and formula collagen for melt spinning as 120d/68f cooling collagen Partial Oriented Yarn (POY), then make a 70d/68f cooling collagen Draw Textured Yarn (DTY) through drawing and false twist processes. Finally, single jersey and double face knitted fabrics with cooling and collagen were fabricated by $32 \mathrm{G}$ and 28G circular machine. The spring and summer women's underwear and casual wear are designed as the product, and the related physical and chemical properties (washing dyeing fastness test faded and stained, shrinkage rate, free formaldehyde content, etc.) and functional properties (instant cool heat flow, UPF, static thermal image, water absorption height, 40-minute residual moisture rate, water absorption area, etc.). The experimental results show that the physical and functional properties can meet the basic requirements of women's underwear and casual wear in spring and summer. The development of spring and summer women's underwear and casual wear is in line with the industrial upgrading and transformation and industry innovation research and development theme, including the combination of upstream (functional fiber), midstream (yarn, fabric), downstream (dyeing and finishing) industry and women's products to integrate cooperated supply chain. Textile Enterprise Co., Ltd. is the market leader in the international underwear knitwear market and combined with a number of international brands to promote this underwear and casual apparel international market which have multi-tone. moisture keeping, cooling feeling, beauty and anti-UV functions.
\end{abstract}

Keywords: DTY with Multi-tone; Multi-functional and beauty use; Circular knitted fabrics; Qmax; UPF; Static Thermal Image; Water absorption ability

\section{Introduction}

In recent years, the research on cooling comfort and collagen textiles is mainly in the field of underwear and casual wear. The collagen is more important in the fields of health food, cosmetics, medicine and man-made fiber. In the field of collagen textile fiber production in recent years, the additive is mainly composed of papolis, collagen extracted from milkfish scales, bone-extracted collagen, and artificial collagen [1]. Collagen is a natural substrate in the natural ecology, which has the following three aspects of health effects on the human body.

1. Comfort - collagen is composed of 18 kinds of amino acids, which has excellent biocompatibility with the human body.

2. It has humidity regulation function, because collagen is rich in hydrophilic groups such as many amine groups $(-\mathrm{CONH})$ and amino groups (-NH2), the structure is porous, easy to diffuse water molecules, and can be adjusted in humid air as well as have a certain amount of water.
3. Tryptophan and Tyrosine in collagen absorb ultraviolet rays, and ultraviolet rays are harmful to the skin, so collagen can protect the skin from ultraviolet light radiation [2-7].

Although the above description shows that collagen has so many advantages and functions, there are few reports and paper on the application of collagen in viscose rayon staple fibers and nylon 6 filament yarns. There is no clear explanation on how to overcome the heat resistance of collagen. Collagen detection cannot be performed quantitatively even in yarns and fabrics. Since the activity and heat resistance of collagen may be destroyed during master batch, spinning, dyeing, and setting stages, the functionality of the fiber products was affected. Due to the problems of collagen yellowing, heat resistance, mold and activity loss caused by high temperature production, this paper uses the special collagen formula and the in-situ polymerization method of the innovative process to overcome the above [8] problems and enhance the performances of collagen nylon 6 fibers such as heat resistance, durability, moisture retention, 


\section{Current Trends in Fashion Technology \& Textile Engineering}

skin-friendly and comfort, etc., and have higher added value and economic benefits.

\section{Experiment}

The fabrication of polyamie 6 with collagon and cooling is made from in-situ polymerization and through melt spinning methos to produce the Partial Oriented Yarns (POY) with $120 d / 68 f$. Furthermore, the 70d/68f Draw Textured Yarn (DTY) was made from Murata nip twister false twist machine [8]. Table 1 shows the DTY content, density, fabric weight and width of three different collagens woven by a 28G, 32G circular knitting machine made of 70d/68f collagen PA6 extended false twisted yarn (DTY). The finished fabric is finished by scouring, bleaching, pre-setting, dyeing, finishing and heat setting processes, and the physical and functional properties described herein are all cool collagen finished fabrics.

Table 1: The specifications and compositions of cooling collagen circular knitting fabrics.

\begin{tabular}{|c|c|c|}
\hline Fabric Code (FC) & FC 32781 & FC 232215 \\
\hline Knitted Machine Gauge & $28 \mathrm{G}$ & $32 \mathrm{G}$ \\
\hline \multirow{2}{*}{ Fabric weight } & $225 \mathrm{~g} / \mathrm{yd}$ & $220 \mathrm{gsm}$ \\
\hline Fabric Width & $158 \mathrm{gsm}$ & $60 \mathrm{inches}$ \\
\hline Thickness & 60 inches & $0.585 \mathrm{~mm}$ \\
\hline Cooling collagen yarn content & $0.455 \mathrm{~mm}$ & $84.00 \%$ \\
\hline
\end{tabular}

\section{Results and Discussion}

\section{Comparison of physicochemical properties of cooling collagen circular knitted fabric}

Table 2: The specifications and compositions of cooling collagen circular knitting fabrics.

\begin{tabular}{|c|c|c|c|}
\hline Testing items & Testing Standard & \multicolumn{2}{|c|}{ Actual Value } \\
\hline Washable dyeing fastness test fades and stains & CNS1494 L3027 & WD & $-0.80 \%$ \\
\hline Fabric Shrinkage & CNS 8038 L31138 & FD & $-0.80 \%$ \\
\hline Free formaldehyde content & CNS 12943 & $\leq 20 \mathrm{ppm}$ & \\
\hline
\end{tabular}

Notes: WD: Warp Direction; FD: Filling Direction

Table 2 shows the results of the fading and staining, shrinkage and free formaldehyde content of cooling collagen knitted fabrics with different gauges. The measured values in Table 2

\section{The functional properties of circular knitted fabrics}

Table 3: The Functional Properties Testing Results of Circular Knitted Fabrics

\begin{tabular}{|c|c|c|c|c|}
\hline Testing Items & Testing Standard & & FC 32781 & FC 232215 \\
\hline Air permeability & ASTM D 737 & & $0.2 \mathrm{~cm}^{3} / \mathrm{cm}^{2} / \mathrm{s}$ & $44.1 \mathrm{~cm}^{3} / \mathrm{cm}^{2} / \mathrm{s}$ \\
\hline Water Absorption Area & \multirow{4}{*}{ FTTS-FA-004 [9] } & \multicolumn{2}{|c|}{ Grade $4\left(1174 \mathrm{~mm}^{2}\right)$} & Grade $4\left(1301 \mathrm{~mm}^{2}\right)$ \\
\hline Water Absorption Height & & WD & Grade $3(97 \mathrm{~mm})$ & Grade $3(95 \mathrm{~mm})$ \\
\hline \multirow{2}{*}{40 Minutes Residual Moisture Rate } & & FD & Grade $4(100 \mathrm{~mm})$ & Grade $3(99 \mathrm{~mm})$ \\
\hline & & \multicolumn{2}{|c|}{ Grade $2(38.50 \%)$} & Grade $2(41.8 \%)$ \\
\hline Q-max & FTTS-FA-019[11] & & $1840 \mathrm{~W} / \mathrm{cm}^{2}$ & $0.200 \mathrm{~W} / \mathrm{cm}^{2}$ \\
\hline Anti-ultraviolet light (UPF) & AATCC 183 & & 229 & 109 \\
\hline Static Thermal Image Temperature & FTTS -FA-010[10] & & $2.2^{\circ} \mathrm{C}$ & $0.8^{\circ} \mathrm{C}$ \\
\hline
\end{tabular}

The functional properties of the two circular knitting fabrics with different gauges and knitted fabric weights are shown in Table 3. The air permeability of the knitted fabrics is gradually increased as the fabric weight and density increase according to the ASTM D 737 testing standard. Since both fabrics have inlaid an elastic fiber (Spandex fiber) about $16 \mathrm{wt} . \%$, there is an elastic recovery rate of more than $120 \%$, so there is no fear in air permeability and wearing comfort problem. The FTTS-FA-004 test specification was draw up Functionality and Industrial Textiles Certification and Verification Review Committee in Taiwan. According to the FTTS-FA-004 test specification, the can reach the spring and summer females basic requirements for international brands of underwear and casual wear. water absorption area, water absorption height and residual moisture content of 40 minutes show that the higher water absorption area has a higher water absorption performance with the increase of fabric weight and the amount of cooling collagen yarn. In the anti-ultraviolet light according to the AATCC 183 test standard, as the weight and density of the fabric increase, the anti-ultraviolet resistance of the knitted fabric gradually increases. In the static thermal image temperature difference according to the FTTS-FA-010 test specification, as the fabric weight and density increase, the thermal image temperature difference of knitted fabrics gradually increases. The multiple 
functions meet the basic requirements of women's underwear and casual wear in spring and summer. Furthermore, the instant cooling feeling value (Qmax) of the FC 232215 knitted fabric better than the nomenclature FC 32781. It is probably because that the higher cooling collagen yarn content has better performance of the knitted fabric.

\section{Conclusion}

The multi-functional and cosmetic fiber was developed successfully in this paper which in response to the Kyoto Protocol and a number of international climate conference thinking regarding energy conservation, year-on-year reduction of carbon emissions and carbon. The single jersey and double face knitted fabrics were fabricated by $32 \mathrm{G}$ and $28 \mathrm{G}$ circular machine. The spring and summer women's underwear and casual wear are designed as the product, and the related physical and chemical properties (washing dyeing fastness test faded and stained, shrinkage rate, free formaldehyde content, etc.) and functionality (instant cool heat flow, UPF, static thermal image, water absorption height, 40-minute residual moisture rate, water absorption area, etc.). The experimental results show that the physical and functional properties can meet the basic requirements of women's underwear and casual wear in spring and summer. The development of spring and summer women's underwear and casual wear is in line with the industrial upgrading and transformation and industry innovation research and development theme, including the combination of upstream (functional fiber), midstream (yarn, fabric), downstream (dyeing and finishing) industry and women's products to integrate cooperated supply chain. Textile Enterprise Co., Ltd. is the market leader in the international underwear knitwear market and combined with a number of international brands to promote this underwear and casual apparel international market which have multi-tone. collagen beauty and anti-UV functions [9-11].

\section{Acknowledgement}

Much appreciated to the Ministry of Science and Technology of the Executive Yuan in Taiwan for providing financial support for this project (E10700010002-015-1). This industrialacademicals project is jointly developed by Textile Enterprise Co., Ltd. and Textile and Materials Industry Research Center of the Feng Chia University. During the implementation of this project, we are very grateful to the multi-tone, multi-functional and beauty fiber supply chain manufacturers for their assistance in mass production and finished product design and testing method of knitted fabrics. We would also like to thank the students from the Organic and Inorganic Composites Laboratory of Department of Fiber and Composite Materials of Feng Chia University and the colleagues of Textile and Materials Industry Research Center for their assistance in testing and polymerization experiments.

\section{References}

1. Donghe Textile Co., Ltd. Moisture Absorption and Dehumidification Testing Report of Papolis Fiber.

2. https: / / tw.answers.yahoo.com/question / index?qid=20050804000012KK18991\&p=\%E8\%A0\%B6\%E7\%B5\%B2\%E 8\%9B\%8B\%E7\%99\%BD

3. Wikipedia $\sim$ Amino Acid.

4. Wikipedia $\sim$ Peptide.

5. Moisturizing factor.

6. http://www.ftts.org.tw/standard.aspx-FTTS-FA-004

7. Fiber Division II, Taiwan Chemical Company, Fish Scale Collagen Nylon Fiber Testing Report.

8. JP Ching (2016) A Study on the PA6/ Cooling Composite Mineral Stone Chips by Using In-Situ Polymerization Method, Master thesis, Department of Fiber and Composite Materials, Feng Chia University, Taiwan.

9. The Committee for Conformity Assessment of Accreditation and Certification on Functional and Technical Textiles (2003), Specified Requirements for Moisture Transferring and Quick Drying Textiles, FTTS-FA-004.

10. The Committee for Conformity Assessment of Accreditation and Certification on Functional and Technical Textiles (2004), Specified Requirements for Far Infrared Textiles, FTTS-FA- 010.

11. The Committee for Conformity Assessment of Accreditation and Certification on Functional and Technical Textiles (2008), Specified Requirements for Cool Feeling Textiles, FTTS-FA-019.

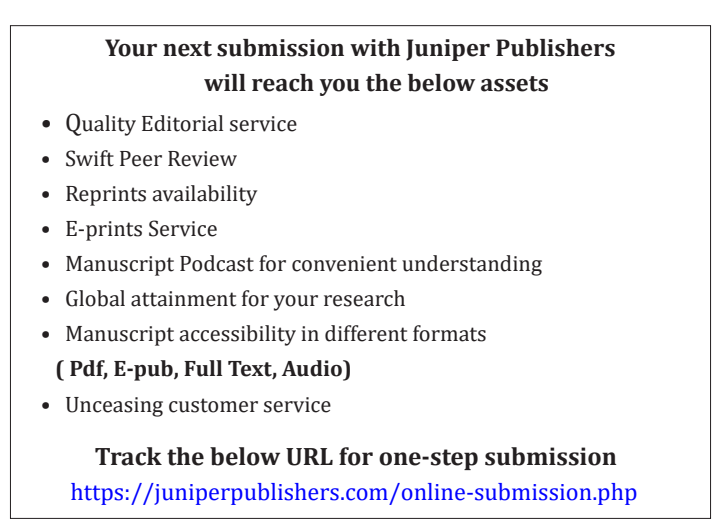

